\title{
Caracterização Florística de Bancos de Sementes em Sistemas De Cultivo Lavoura-Pastagem ${ }^{1}$
}

\author{
Floristic Characterization of Seedbanks Under Integrated Crop-Pasture Systems
}

\author{
IKEDA, F.S. ${ }^{2}$, MITJA, D. ${ }^{3}$, CARMONA, R. ${ }^{4}$ e VILELA, L. ${ }^{5}$
}

\begin{abstract}
RESUMO - Avaliou-se o efeito de sistemas de cultivo, preparo do solo e níveis de adubação sobre a composição florística de banco de sementes no solo em áreas submetidas a três sistemas de cultivo (lavoura contínua, lavoura-pastagem-lavoura e pastagem-lavourapastagem), dois sistemas de preparo do solo (convencional e semeadura direta) e dois níveis de adubação (manutenção e corretiva gradual), e uma área de pastagem contínua com preparo convencional e adubação corretiva gradual. Dessa forma, verificou-se que o sistema de cultivo e o sistema de preparo do solo foram os fatores mais importantes na determinação da estrutura florística dos bancos de sementes. Além disso, a adubação corretiva gradual aumentou o número de famílias e de espécies, em relação à de manutenção.
\end{abstract}

Palavras-chave: adubação, Cerrado, planta daninha, semeadura direta.

\begin{abstract}
The effect of cultivation systems, tillage and fertilization levels on the floristic composition of seedbanks was evaluated at three cultivation systems (continuous crop, crop-pasturecrop, pasture-crop-pasture), two tillage systems (tillage and no-tillage) and two fertilization levels (corrective and maintenance), and a continuous pasture area under no-tillage and corrective fertilization. Cultivation and tillage system were the most important factors affecting the floris tic structure of seedbanks. Besides, corrective fertilization increased the number of families and species in relation to maintenance fertilization.
\end{abstract}

Keywords: fertilization, Cerrado, weed, no-tillage.

\section{INTRODUÇÃO}

A integração dos sistemas de produção de grãos e de pecuária vem ganhando força como alternativa para os problemas advindos das lavouras e pastagens contínuas. Essa integração pode reduzir os riscos de degradação do solo, melhorando suas propriedades químicas, físicas e biológicas e aumentando o potencial produtivo de grãos e de forragem (Vilela et al., 2003). Na integração de sistemas de produção, existe a possibilidade de aplicar diversas combinações de culturas anuais e pastagens, além de ser possivel variar a intensidade de preparo do solo, manejo da fertilidade e das práticas de controle de plantas daninhas. Essas combinações normalmente afetam o banco de sementes de plantas daninhas no solo em áreas de lavoura contínua (Cardina et al., 2002; Légère et al., 2005). No entanto, praticamente não há estudos relacionados aos sistemas de cultivo lavoura-pastagem. Nas áreas de pastagem continua ocorrem geralmente espécies nativas (Nunes, 2001) e menor número de

Recebido para publicação em 28.3.2007 e na forma revisada em 25.10.2007.

2 Eng-Agra -, M.Sc., Embrapa Cerrados, BR $020 \mathrm{~km} 18$ - Caixa Postal 08223, 73310-970, Planaltina, DF, $<$ fernanda.satie.ikeda@gmail.com>; ${ }^{3}$ Bióloga, D.Sc., Pesquisadora do Institut de Recherche pour le Dévelopement, $<$ danielle.mitja@ird.fr $>$; ${ }^{4}$ Eng $^{\circ}$-Agr ${ }^{\circ}$., Ph.D., Prof. Titular da Faculdade de Agronomia e Medicina Veterinária, UnB $<$ rcarmona@unb.br>; ${ }^{5}$ Eng $^{\mathrm{e}}$-Agr ${ }^{\circ}$., M.Sc., Pesquisador da Embrapa Cerrados, <lvilela@cpac.embrapa.br>.

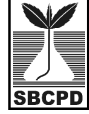

Planta Daninha, Viçosa-MG v. 25, n. 4, p. 735-745, 2007 
espécies do que em áreas de lavoura (Gasparino et al., 2006), o que pode contribuir para modificação da estrutura florística dos bancos de sementes em áreas de lavoura que sucedem o cultivo de pastagens. Nas áreas de lavoura contínua, observa-se uma tendência ao predomínio de espécies dicotiledôneas de ciclo anual com o preparo convencional e de espécies perenes, gramíneas anuais e espécies disseminadas pelo vento com o sistema de cultivo mínimo (Froud-Willians et al., 1981).

O estudo da estrutura florística dos bancos de sementes permite observar quais famílias e espécies assumem maior importância na composição desses bancos. Para isso, o método de plântulas emergidas em casa de vegetação (Ball \& Miller, 1989) possibilita a avaliação das espécies que compõem o banco de sementes (Sosnoskie et al., 2006). Por meio de análises multivariadas, como a classificação de espécies, é possivel verificar quais são os fatores mais importantes na determinação da composição de bancos de sementes e as espécies que estão mais correlacionadas a esses fatores (Kent \& Coker, 1992).

O presente trabalho objetivou avaliar o efeito de diferentes sistemas de cultivo, incluindo o preparo do solo e o nível de adubação, na estrutura florística dos bancos de sementes do solo.

\section{MATERIAL E MÉTODOS}

\section{Área Experimental}

Este trabalho foi conduzido em áreas integrantes de um experimento de cultivo lavourapastagem instalado em 1991, na Embrapa Cerrados, Planaltina, DF. A área experimental localiza-se em Latossolo Vermelho, textura argilosa, entre as coordenadas de $15^{\circ} 39^{\prime} 84^{\prime \prime} \mathrm{S} \mathrm{e}$ $47^{\circ} 44^{\prime} 41^{\prime \prime} \mathrm{W}$, em altitude aproximada de $1.217 \mathrm{~m}$. O clima no local foi classificado como Aw (Köppen), com duas estações bem definidas (seca e chuvosa) e a ocorrência de períodos de estiagem durante a estação chuvosa (veranicos). A área experimental foi planejada e conduzida com o objetivo de avaliar combinações de culturas e pastagens, acompanhando as tendências no uso de forrageiras e de manejo cultural no sistema de cultivo lavoura-pastagem.

Planta Daninha, Viçosa-MG v. 25, n. 4, p. 735-745, 2007
Foram selecionadas 12 áreas desse experimento, submetidas a três sistemas de cultivo (lavoura continua "L", lavoura-pastagem-lavoura "LPL" e pastagem-lavoura-pastagem "PLP"), dois sistemas de preparo do solo (convencional " $C$ " e semeadura direta " $D$ ") e dois níveis de adubação (manutenção "1" e corretiva gradual “2”). Avaliou-se também uma área de pastagem continua ("P") com preparo convencional e adubação corretiva gradual. A dimensão de cada área foi de 40 x $100 \mathrm{~m}$.

O histórico das áreas contendo a seqüência de culturas e o total de calcário e de fertilizantes aplicados nos dois niveis de adubação de cada sistema de cultivo encontra-se descrito na Tabela 1. Os ciclos de lavoura ou de pastagem nos sistemas de cultivo lavoura-pastagem ocorreram nas safras de 1991/1992 a 1994/ $1995,1995 / 1996$ a 1998/1999 e 1999/2000 a $2003 / 2004$. Houve rotação entre culturas de grãos nas lavouras contínuas e nas fases de lavoura dos sistemas de cultivo lavoura-pastagem. As forrageiras utilizadas nas fases de pastagem dos sistemas de cultivo lavoura-pastagem e na pastagem contínua foram substituídas a partir das safras de 1999/2000 e 2000/2001, respectivamente. A renovação das pastagens tinha como finalidade aproveitar a melhoria da fertilidade, com a introdução de forrageiras mais produtivas e propícias para o período de seca do que o Andropogon gayanus.

Nas áreas de lavoura, o preparo convencional do solo foi efetuado mediante uma gradagem pesada após a colheita e uma gradagem pesada seguida por gradagem de nivelamento antes da semeadura. A semeadura direta foi estabelecida a partir da safra de 1995/1996, com dessecação prévia de plantas daninhas. Na safra de 2003/2004 foi semeada Brachiaria brizantha como planta de cobertura apenas nas áreas de cultivo de LPLD, com o objetivo de formar a cobertura morta da semeadura direta no ano seguinte, de acordo com o sistema Santa Fé, descrito por Cobucci \& Portela (2003).

Os tratos culturais foram aplicados de acordo com as recomendações de cada cultura. Nos anos de cultivo da soja, foram utilizados os herbicidas trifluralin e imazaquin em preparo convencional para aplicações em pré-emergência e o glyphosate para controle da vegetação em pré-plantio em semeadura direta. Em ambos os sistemas de preparo de solo foram 
Tabela 1 - Histórico de culturas e total de calcário e fertilizantes aplicados nos sistemas de cultivo em experimento de cultivo lavoura pastagem - Embrapa Cerrados, Planaltina-DF

\begin{tabular}{|c|c|c|c|c|c|c|c|c|c|c|c|c|c|}
\hline \multirow{3}{*}{$\begin{array}{c}\text { Sistema } \\
\text { de cultivo }\end{array}$} & \multicolumn{3}{|c|}{ Cultura } & \multicolumn{5}{|c|}{ Adubação de manutenção } & \multicolumn{5}{|c|}{ Adubação corretiva gradual } \\
\hline & \multicolumn{3}{|c|}{ Ciclos } & Calcário & $\mathrm{N}$ & $\mathrm{P}_{2} \mathrm{O}_{5}$ & $\mathrm{~K}_{2} \mathrm{O}$ & Gesso & Calcário & $\mathrm{N}$ & $\mathrm{P}_{2} \mathrm{O}_{5}$ & $\mathrm{~K}_{2} \mathrm{O}$ & Gesso \\
\hline & $91 / 92-94 / 95$ & 95/96-98/99 & $99 / 00-03 / 04$ & $\mathrm{t} \mathrm{ha}^{-1}$ & \multicolumn{3}{|c|}{$\mathrm{kg} \mathrm{ha}^{-1}$} & $\mathrm{t} \mathrm{ha}^{-1}$ & $\mathrm{t} \mathrm{ha}^{-1}$ & \multicolumn{3}{|c|}{$\mathrm{kg} \mathrm{ha}^{-1}$} & $\mathrm{t} \mathrm{ha}^{-1}$ \\
\hline $\mathrm{L}$ & $\mathrm{S} / \mathrm{S} / \mathrm{M} / \mathrm{S}$ & $\mathrm{M} / \mathrm{S} / \mathrm{M} / \mathrm{S}$ & $\mathrm{S} / \mathrm{Mi} / \mathrm{S} / \mathrm{Mi} / \mathrm{S}$ & 4,4 & 120 & 594 & 466 & 0,0 & 7,5 & 235 & 1123 & 1037 & 2,8 \\
\hline LPL & $\mathrm{S} / \mathrm{S} / \mathrm{M} / \mathrm{S}$ & $\mathrm{A} / \mathrm{A} / \mathrm{A} / \mathrm{A}$ & $\mathrm{S} / \mathrm{Mi} / \mathrm{S} / \mathrm{Mi} / \mathrm{S}+\mathrm{B} *$ & 4,4 & 40 & 406 & 318 & 0,0 & 7,5 & 85 & 768 & 723 & 2,8 \\
\hline PLP & $\mathrm{A} / \mathrm{A} / \mathrm{A} / \mathrm{A}$ & $\mathrm{M} / \mathrm{S} / \mathrm{M} / \mathrm{S}$ & $\mathrm{P} / \mathrm{P} / \mathrm{P} / \mathrm{P} / \mathrm{P}$ & 4,4 & 120 & 327 & 207 & 0,0 & 7,5 & 190 & 511 & 380 & 0,0 \\
\hline $\mathrm{P}$ & $\mathrm{A} / \mathrm{A} / \mathrm{A} / \mathrm{A}$ & $\mathrm{A} / \mathrm{A} / \mathrm{A} / \mathrm{A}$ & $\mathrm{A} / \mathrm{Bd} / \mathrm{Bd} / \mathrm{Bd} / \mathrm{Bd}$ & - & - & - & - & - & 6,8 & 80 & 254 & 204 & 0,0 \\
\hline
\end{tabular}

$\mathrm{L}=$ lavoura contínua; $\mathrm{LPL}=$ lavoura-pastagem-lavoura; $\mathrm{PLP}=$ pastagem-lavoura-pastagem; $\mathrm{P}=$ pastagem contínua; $\mathrm{A}=$ capimandropógon (Andropogon gayanus); B* = braquiarão (Brachiaria brizantha) apenas na semeadura direta; Bd = capim-braquiária (B. decumbens); $\mathrm{M}=$ milho (Zea mays); $\mathrm{Mi}=$ milheto (Pennisetum americanum); $\mathrm{P}=$ capim-tanzânia (Panicum maximum cv. Tanzânia); S = soja (Glycine max).

usados os herbicidas haloxyfop-methyl, fluazifop-p-butyl + fomesafen, fluazifop-p-butyl, metolachlor, diclosulam ou chlorimuron-ethyl, dependendo da necessidade. Nas áreas de cultivo de LPLD, foram aplicadas doses reduzidas de haloxyfop-methyl para controlar o crescimento de $B$. brizantha, de forma a reduzir a competição com a soja. Nos anos de cultivo do milho foram utilizados os herbicidas atrazine + metolachlor. Não foram utilizados herbicidas no cultivo de milheto. As doses utilizadas seguiram as recomendações de cada produto.

Nas pastagens contínuas, o preparo convencional foi efetuado apenas na implantação (1991) e na renovação (2000) das pastagens. Nas áreas de cultivo de PLP, o preparo ocorreu na fase de lavoura e antes do estabelecimento das pastagens. Nas pastagens contínuas, as adubações foram feitas de acordo com as recomendações para as espécies, com base nos resultados de análise do solo e de produtividade esperada. No sistema de cultivo lavoura-pastagem, a adubação residual das lavouras permitiu o estabelecimento das pastagens. Não foram utilizados herbicidas nas pastagens.

\section{Amostragem de Solo}

A amostragem do solo para avaliação do banco de sementes foi efetuada na última safra do ciclo de 1999/2000-2003/2004, depois da colheita da soja e antes do preparo para a safra seguinte. Realizou-se a coleta entre os meses de outubro e novembro de 2004, com duração de um mês. Adotou-se uma amostragem sistemática por conglomerados, com coleta de oito amostras compostas por quatro subamostras com diâmetro de $7,3 \mathrm{~cm}$. As coletas foram realizadas em duas linhas transversais às de cultivo, nas profundidades de 0 a $5 \mathrm{~cm}$ e de 5 a $20 \mathrm{~cm}$, totalizando 104 amostras para cada profundidade. A distância entre os pontos de amostragem foi de $10 \mathrm{~m}$, desconsiderando-se $5 \mathrm{~m}$ de bordadura.

\section{Método de Avaliação}

Depois da secagem ao ar, homogeneizaram-se manualmente as amostras de solo de 0 a $5 \mathrm{~cm}$. Houve necessidade de homogeneizar melhor as amostras de 5 a $20 \mathrm{~cm}$ por meio de um divisor de solos, após a quebra de torrões em peneira. Esse procedimento teve como finalidade avaliar de forma mais representativa o número de plântulas emergidas em apenas um terço dessas amostras, pois houve necessidade de reduzir o espaço ocupado em casa telada. Foram utilizadas bandejas plásticas perfuradas de dimensões $19,5 \times 19,5 \times 6,5 \mathrm{~cm}$ para acondicionamento das amostras de solo. Previamente à disposição das amostras de solo, cada bandeja recebeu $0,30 \mathrm{~L}$ de areia esterilizada, para facilitar a drenagem da água e evitar o escoamento das amostras. As bandejas foram distribuídas aleatoriamente dentro de casa telada, incluindo oito bandejas de controle com somente areia esterilizada, para monitoramento de contaminação externa de sementes.

As amostras foram irrigadas diariamente a partir de dezembro de 2004 . Houve presença de luz e alternância de temperaturas na casa telada, de forma que favorecesse a germinação e a quebra de dormência. Após seis meses de 
emergência, avaliação e descarte de plântulas, o solo foi revolvido, em junho de 2005, precedido de cinco dias sem irrigação, para que novos fluxos de emergência pudessem ocorrer. A emergência de plântulas cessou ao final de aproximadamente oito meses de avaliação, totalizando 16 levantamentos, espaçados de aproximadamente 15 dias. O número de sementes foi obtido pela soma das plântulas emergidas em cada amostra durante esse período. Foram coletadas amostras de plântulas, plantas adultas e plantas com flores e/ou frutos, com a finalidade de confirmar a identificação botânica em herbário. Esse procedimento foi efetuado na Embrapa Recursos Genéticos e Biotecnologia e na Reserva Ecológica do IBGE. As amostras com flores e/ou frutos foram depositadas no Herbário do IBGE.

\section{Análise de Dados}

Avaliou-se o número de famílias e espécies nas profundidades de 0 a 5,5 a 20 e 0 a $20 \mathrm{~cm}$ de cada área. Os dados foram expressos em sementes por $\mathrm{m}^{2}$. Para identificar as famílias e as espécies mais características e importantes das áreas, utilizaram-se os agrupamentos de espécies com dispersão semelhante entre os grupos de áreas formados pelo TWINSPAN (two-way species indicator analysis). Além disso, foi calculado o índice de valor de importância (IVI) de cada família e espécie na profundidade de 0 a $20 \mathrm{~cm}$ de cada área. A classificação por TWINSPAN foi efetuada no programa PC-ORD 3.17, utilizando uma matriz de dados de presença/ausência das espécies encontradas na profundidade de 0 a $20 \mathrm{~cm}$ das 13 áreas avaliadas. O TWINSPAN faz uma classificação hierárquica das espécies em função das parcelas onde elas ocorrem e das parcelas em função das espécies que nelas ocorrem. A classificação divide um grupo de áreas ou espécies em dois grupos, cada um deles em outros dois grupos, e assim sucessivamente, sendo o último nível de divisão definido pelo usuário (Kent \& Coker, 1992). Os agrupamentos de áreas propostos pelo TWINSPAN são normalmente conservados. Entretanto, na classificação de espécies em função das áreas, algumas delas geralmente são remanejadas para grupos mais característicos, conforme a ocorrência nas áreas.
O IVI foi obtido pela soma de densidade relativa e freqüência relativa (Mueller-Dombois \& Ellenberg, 1974), da seguinte forma:

$$
\mathrm{IVI}=(\mathrm{DR}+\mathrm{FR})
$$

em que:

$\mathrm{DR}=$ densidade relativa

$$
\mathrm{DR}=\frac{(\mathrm{DA} \times 100)}{\Sigma \mathrm{DA}}
$$

$\mathrm{DA}=$ densidade absoluta (n de sementes de cada espécie $/ \mathrm{m}^{2}$ );

$\mathrm{FR}=$ freqüência relativa

$$
\mathrm{FR}=\frac{(\mathrm{FA} \times 100)}{\Sigma \mathrm{FA}}
$$

$\mathrm{FA}=$ freqüência absoluta de espécies $(\%$ de parcelas em que ocorre cada espécie).

\section{RESULTADOS E DISCUSSÃO}

\section{Classe}

Não ocorreu contaminação externa de sementes de acordo com os resultados das bandejas controle. Em todas as áreas, houve predomínio de espécies magnoliopsidas (dicotiledôneas), com maior IVI e número de famílias e espécies. Apesar de apresentarem número menor de famílias, as liliopsidas (monocotiledôneas) tiveram importância considerável em relação ao número de espécies e IVI. Isso se deve, em grande parte, à família Poaceae (Tabela 2).

\section{Familia}

Foram encontradas 16 famílias na profundidade de 0 a $20 \mathrm{~cm}$ nas 13 áreas avaliadas (Tabela 2). A classificação das áreas em ordem crescente quanto ao número de famílias foi a seguinte: PLPC1 (6) < LPLC1 = LPLD1 = PLPD1 $(7)<\mathrm{LC} 1=\mathrm{LC} 2=\mathrm{PLPD} 2=\mathrm{PC} 2(8)<\mathrm{LD} 1=$ PLPC2 (9) < LD2 = LPLC2 = LPLD2 (11) (Tabela 2). De acordo com esses resultados, verificou-se que as áreas com adubação de manutenção em sistemas de cultivo lavoura-pastagem apresentaram menor número de 


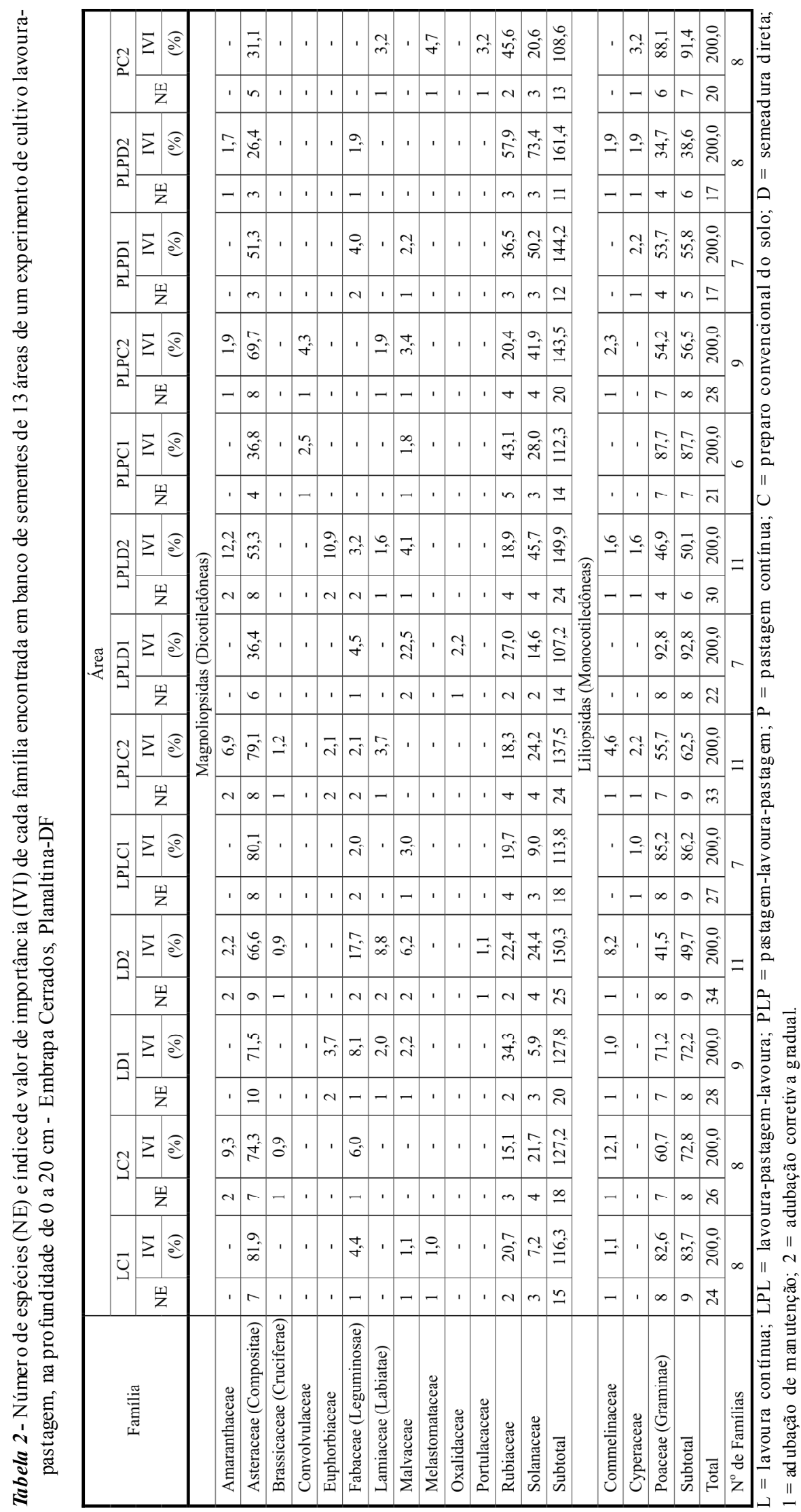


famílias (6 e 7). As áreas de lavoura com adubação corretiva gradual (cultivo de LPL e lavoura contínua com semeadura direta) apresentaram maior número de famílias (11).

Houve menor número de familias na profundidade de 0 a $5 \mathrm{~cm}$ do que na de 5 a $20 \mathrm{~cm}$ nas áreas de cultivo PLPC2, PLPD1 e PLPD2, mesmo número de famílias nas áreas de cultivo LC1 e PLPC1 e maior número de famílias na profundidade de 0 a $5 \mathrm{~cm}$ nas demais áreas (Tabela 3). As famílias com maior IVI nas áreas foram: Asteraceae (LC2, LD1, LD2, LPLC2, LPLD2 e PLPC2), Solanaceae (PLPD2) e Poaceae (LC1, LPLC1, LPLD1, PLPC1, PLPD1 e PC2) (Tabela 2). O maior IVI dessas famílias se deve em parte ao maior número de espécies que apresentaram. De acordo com Kissmann \& Groth (1999), a familia Asteraceae apresenta excepcional importância como infestante, pelo grande número de espécies (20.000 a 25.000) e pela agressividade que em geral apresenta. Da mesma forma, segundo Kissmann (1997), há grande número de espécies entre as Poaceae.

As principais famílias apresentaram condições ambientais preferenciais para o desenvolvimento. Ao avaliar a importância das famílias em relação à cultura presente na área, verificou-se que as Asteraceae apresentaram maior indice de valor de importância nas áreas com lavoura (de 66,6 a 81,9\%) do que nas áreas com presença de forrageiras, incluindo-se as áreas de LPLD (de 26,4 a 69,7\%). Isso, talvez, por causa da necessidade de condições semelhantes às da soja para o desenvolvimento das Asteraceae (Tabela 2). Ao contrário, as Solanaceae foram mais importantes nas áreas com forrageiras (de 14,6 a 73,4\%) do que nas lavouras (de 5,9 a 24,4\%), devido, provavelmente, ao fato de muitas espécies da família Solanaceae apresentarem comportamento de plantas pioneiras em ambientes alterados (Kissmann, 2000). As Poaceae apresentaram valores de IVI semelhantes entre as áreas de lavoura (de 41,5 a $85,2 \%$ ) e áreas com forrageiras (de 34,7 a $92,8 \%$ ), possivelmente em razão da capacidade de adaptação de grande parte das espécies pertencentes à família (Kissmann, 1997). Em relação ao preparo do solo, a família Asteraceae apresentou valor de IVI de 74,3 a $81,9 \%$ em áreas de lavoura com preparo convencional e de 26,4 a $71,5 \%$ em áreas com semeadura direta ou pastagem (considerando-se que não houve praticamente revolvimento do solo nas pastagens). Esse resultado difere daquele observado por Tuesca et al. (2001), em que espécies disseminadas pelo vento apresentaram maior densidade de sementes com a semeadura direta do que com o preparo convencional. Na Tabela 2, observou-se também que as Poaceae foram mais importantes em áreas com adubação de manutenção e as Solanaceae, em áreas com adubação corretiva gradual. As Asteraceae apresentaram importância semelhante nos dois niveis de adubação.

Embora o manejo das áreas tenha influenciado a estrutura florística dos bancos de sementes, deve-se observar que algumas familias estavam presentes nas mais diversas condições, como Asteraceae, Rubiaceae, Solanaceae e Poaceae, que ocorreram em todas as áreas, ao contrário de Oxalidaceae, Convolvulaceae, Melastomataceae e Portulacaceae, que ocorreram em um número restrito de áreas, o que poderia caracterizar maior capacidade de dispersão e/ou reprodução do primeiro grupo de famílias. As Euphorbiaceae e Oxalidaceae ocorreram apenas na profundidade de 0 a $5 \mathrm{~cm}$. Esse resultado condiz com o de Vargas et al. (1999), que observaram taxa de germinação significativamente reduzida de Euphorbia heterophylla com o aumento da profundidade do solo, podendo-se tornar uma planta daninha importante em áreas com semeadura direta ou cultivo mínimo.

\section{Espécie}

Foram encontradas 64 espécies na profundidade de 0 a $20 \mathrm{~cm}$ nas 13 áreas avaliadas, considerando-se que o gênero Pennisetum spp. (com três espécies) foi contabilizado como apenas uma espécie. Houve certa relação entre o número de famílias e o de espécies nos diversos sistemas. Assim, a classificação das áreas em ordem crescente quanto ao número de espécies ocorrentes foi a seguinte: PLPD1 = PLPD2 $(17)<$ PC2 $(20)<$ PLPC1 $(21)<$ LPLD1 (22) < LC1 $(24)<$ LC2 $(26)<$ LPLC1 $(27)<$ LD1 = PLPC2 (28) $<$ LPLD2 (30) < LPLC2 (33) < LD2 (34) (Tabela 2). De acordo com essa classificação, as áreas com forrageiras apresentaram menor número de espécies em relação às áreas de lavoura, excluindo-se as áreas de LPLD2 e PLPC2, que 
estavam entre aquelas com maior número de espécies, juntamente com as áreas LD2 e LPLC2. Além da presença de forrageiras, que, em geral, proporcionam menor número de espécies que as lavouras (Gasparino et al., 2006), esses resultados também estariam associados ao maior número de espécies que a adubação corretiva gradual estaria proporcionando em relação à adubação de manutenção. Além disso, houve maior número de espécies na profundidade de 0 a $5 \mathrm{~cm}$ do que na de 5 a $20 \mathrm{~cm}$ na maioria das áreas, enquanto nas áreas de cultivo de PLPD praticamente não houve diferença entre as duas profundidades (Tabela 3). Esses resultados diferem em parte daqueles obtidos por Cardina et al. (2002), que encontraram maior número de espécies na profundidade de 0 a $5 \mathrm{~cm}$ do que nas de 5 a 10,10 a 15 e 15 a $20 \mathrm{~cm}$ em áreas de lavoura com semeadura direta. Entretanto, não observaram diferença no número de espécies entre as profundidades avaliadas em áreas de lavoura com preparo convencional. Da mesma maneira que as famílias, várias espécies apresentaram condições ambientais preferenciais para o desenvolvimento, conforme constatado na classificação por TWINSPAN e na análise de IVI.

As es pécies foram classificadas por TWINSPAN de acordo com a sua dispersão nas áreas, formando sete conjuntos florísticos distribuídos em três grupos de áreas. A primeira divisão das áreas apresentou autovalor de 0,210, separando as áreas PC2, PLPD1 e PLPD2 (grupo 3) das demais áreas. A segunda divisão apresentou autovalor de 0,182, separando as demais áreas entre o grupo 1 e o grupo 2 . O grupo 1 (LD1, LD2, LPLD1 e LPLD2), correspondente às áreas de lavoura com semeadura direta, foi constituído pelos conjuntos florísticos SPP 1, 2, 4 e 6 de espécies (Tabela 4). Já o grupo 2 (LC1, LC2, LPLC1, LPLC2, PLPC1 e PLPC2), constituído pelas áreas de lavoura com preparo convencional do solo, foi formado pelos conjuntos florísticos SPP 2 e 4, enquanto o grupo 3 (PLPD1, PLPD2 e PC2), correspondente às áreas de pastagem com baixa perturbação do solo, constituiu-se dos conjuntos florísticos SPP 6 e 7. Cinco dos sete conjuntos florísticos apresentaram espécies que ocorreram em praticamente todas as áreas do(s) grupo(s) a que pertencem. Os outros dois conjuntos (SPP 3 e SPP 5) não puderam ser interpretados de maneira confiável, pois apresentaram espécies com presença em um número restrito de áreas dentro do(s) grupo(s) nos quais foram classificados.

O conjunto SPP 1 foi constituído por espécies que ocorreram somente em lavouras contínuas ou de cultivo de LPL com semeadura direta, e a espécie considerada característica desse conjunto foi Conyza bonariensis, pois ocorreu em todas as áreas (4/4) (Tabela 4). O conjunto florístico SPP 2 foi formado por espécies presentes em áreas de cultivo de PLP com preparo convencional do solo, lavouras contínuas e de cultivo de LPL. Blainvillea rhomboidea e Pennisetum spp. foram as

Tabela 3 - Número de famílias (NF) e de espécies (NE) encontradas em banco de sementes de 13 áreas de um experimento de cultivo lavoura-pastagem, na profundidade de 0 a 5 e de 5 a $20 \mathrm{~cm}$ - Embrapa Cerrados, Planaltina-DF

\begin{tabular}{|c|c|c|c|c|c|c|c|c|}
\hline \multirow{3}{*}{ Sistema } & \multicolumn{4}{|c|}{ Adubação de manutenção } & \multicolumn{4}{|c|}{ Adubação corretiva gradual } \\
\hline & \multicolumn{2}{|c|}{$\mathrm{NF}$} & \multicolumn{2}{|c|}{ NE } & \multicolumn{2}{|c|}{ NF } & \multicolumn{2}{|c|}{ NE } \\
\hline & 0 a $5 \mathrm{~cm}$ & 5 a $20 \mathrm{~cm}$ & 0 a $5 \mathrm{~cm}$ & 5 a $20 \mathrm{~cm}$ & 0 a $5 \mathrm{~cm}$ & 5 a $20 \mathrm{~cm}$ & 0 a $5 \mathrm{~cm}$ & 5 a $20 \mathrm{~cm}$ \\
\hline $\mathrm{LC}$ & 6 & 6 & 22 & 15 & 8 & 7 & 25 & 22 \\
\hline LD & 8 & 5 & 26 & 15 & 10 & 9 & 31 & 18 \\
\hline LPLC & 7 & 6 & 25 & 16 & 11 & 10 & 28 & 23 \\
\hline LPLD & 7 & 6 & 21 & 12 & 11 & 6 & 27 & 11 \\
\hline PLPC & 5 & 5 & 20 & 13 & 6 & 9 & 22 & 18 \\
\hline PLPD & 5 & 7 & 13 & 14 & 5 & 7 & 14 & 15 \\
\hline $\mathrm{PC}$ & - & - & - & - & 7 & 5 & 17 & 8 \\
\hline
\end{tabular}

$\mathrm{L}=$ lavoura contínua; $\mathrm{LPL}=$ lavoura-pastagem-lavoura; $\mathrm{PLP}=$ pastagem-lavoura-pastagem; $\mathrm{P}=$ pastagem contínua; $\mathrm{C}=$ preparo convencional do solo; $\mathrm{D}=$ semeadura direta. 
Tabela 4 - Classificação por TWINSPAN quanto à presença ou ausência de espécies em função do banco de sementes na profundidade de 0 a $20 \mathrm{~cm}$ das áreas avaliadas em experimento de cultivo lavoura-pastagem - Embrapa Cerrados, PlanaltinaDF. Áreas em cinza representam o(s) grupo(s) de áreas em que ocorreram os conjuntos florísticos

\begin{tabular}{|c|c|c|c|c|c|c|c|c|c|c|c|c|c|c|}
\hline \multirow{3}{*}{$\begin{array}{l}\text { Coniunto } \\
\text { Floristico }\end{array}$} & \multirow{3}{*}{ Espécie } & \multicolumn{13}{|c|}{ Área } \\
\hline & & \multicolumn{4}{|c|}{ Grupo 1} & \multicolumn{6}{|c|}{ Grupo 2} & \multicolumn{3}{|c|}{ Grupo 3} \\
\hline & & $\overline{\mathrm{G}}$ & $气$ & 馬 & క్తి & שֶ & $\overrightarrow{\breve{J}}$ & 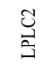 & $\bar{\Xi}$ & $\begin{array}{l}\bar{\Xi} \\
\vdots \\
\vdots\end{array}$ & 䜦 & $\tilde{\Sigma}$ & 产 & 兽 \\
\hline \multirow{8}{*}{ SPP 1} & Andropogon gayanus & & 1 & 1 & & & & & & & & & & \\
\hline & Axonopus capillaris & & & 1 & & & & & & & & & & \\
\hline & Crotalaria sp. & & 1 & & & & & & & & & & & \\
\hline & Cyperus sesquiflorus & & & & 1 & & & & & & & & & \\
\hline & Conyza bonariensis & 1 & 1 & 1 & 1 & & & & & & 1 & & & \\
\hline & Marsypianthes chamaedrys & 1 & 1 & & & & & & & & & & & \\
\hline & Oxalis corniculata & & & 1 & & & & & & & & & & \\
\hline & Phyllanthus tenellus & 1 & & & & & & & & & & & & \\
\hline \multirow{17}{*}{ SPP 2} & Bidens pilosa & 1 & 1 & & 1 & & & 1 & 1 & & & & & \\
\hline & Sida rhombifolia & 1 & 1 & 1 & 1 & & 1 & & & & 1 & & & \\
\hline & Bidens subalternans & 1 & 1 & 1 & 1 & & 1 & 1 & 1 & & & & & \\
\hline & Centratherum ct. camporum & 1 & 1 & 1 & 1 & 1 & 1 & & 1 & & & & & \\
\hline & Chamaesvce hirta & 1 & & & 1 & & & 1 & & & & & & \\
\hline & Stylosanthes guianensis & & & 1 & 1 & & 1 & & & & & & & \\
\hline & Brachiaria plantaginea & & 1 & & & & & & & 1 & & & & \\
\hline & Desmodium sp & 1 & 1 & & & 1 & & & 1 & & & & & \\
\hline & Pennisetum spp. ${ }^{*}$ & 1 & 1 & 1 & . & 1 & 1 & 1 & 1 & & 1 & & & \\
\hline & Solanum sisymbriifolium & & 1 & & 1 & 1 & & 1 & & & 1 & & & \\
\hline & Altcrnanthera tenclla & & 1 & & 1 & 1 & & 1 & & & & & & \\
\hline & Euphorbia heterophylla & & & & 1 & & & 1 & & & & & & \\
\hline & Eupatorium pauciflorum & 1 & & & & & 1 & & & & & & & \\
\hline & Blainvillea rhomboidea & 1 & 1 & & 1 & 1 & 1 & 1 & 1 & 1 & 1 & & & \\
\hline & Lepidium ruderale & & 1 & & & 1 & & 1 & & & & & & \\
\hline & Rhynchelitrum repens & 1 & 1 & & & 1 & 1 & 1 & 1 & 1 & 1 & & & \\
\hline & Emilia sonchifolia & 1 & & & 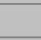 & 1 & 1 & 1 & 1 & 1 & 1 & & & \\
\hline \multirow{17}{*}{ SPP 4} & Spermacoce cf. capitata & & & 1 & 1 & 1 & 1 & 1 & 1 & 1 & 1 & & 1 & \\
\hline & Spermacoce cf. verticilata & & & & 1 & & 1 & 1 & & 1 & 1 & & & 1 \\
\hline & Cenchrus echinatus & 1 & 1 & 1 & & 1 & 1 & 1 & 1 & & & 1 & & \\
\hline & Commelina henghalensis & 1 & 1 & & 1 & 1 & & 1 & 1 & . & 1 & & & 1 \\
\hline & Digitaria $\mathrm{cf}$. horizontalis & 1 & 1 & 1 & 1 & 1 & 1 & 1 & 1 & 1 & 1 & 1 & & \\
\hline & Amaranthus cf. viridis & & 1 & & 1 & 1 & & 1 & & & 1 & & & 1 \\
\hline & Sida glaziovii & & 1 & 1 & & & . & & 1 & 1 & & & 1 & \\
\hline & Digitaria ciliaris & 1 & 1 & 1 & 1 & 1 & 1 & 1 & 1 & 1 & 1 & & 1 & 1 \\
\hline & Acanthospermum australe & 1 & 1 & 1 & 1 & 1 & 1 & 1 & 1 & 1 & 1 & 1 & 1 & 1 \\
\hline & Ageratum conyzoides & 1 & 1 & 1 & 1 & 1 & 1 & 1 & 1 & 1 & 1 & 1 & 1 & 1 \\
\hline & Eleusine indica & 1 & 1 & 1 & 1 & 1 & 1 & 1 & 1 & 1 & 1 & 1 & 1 & 1 \\
\hline & Richardia brasiliensis & 1 & 1 & 1 & 1 & 1 & 1 & 1 & 1 & 1 & 1 & 1 & 1 & 1 \\
\hline & Schwenckia americana & 1 & 1 & 1 & 1 & 1 & 1 & 1 & 1 & 1 & 1 & 1 & 1 & 1 \\
\hline & Solanum americanum & 1 & 1 & 1 & 1 & 1 & 1 & 1 & 1 & 1 & 1 & 1 & 1 & 1 \\
\hline & Erugrostis rufescens & 1 & & 1 & 1 & 1 & 1 & 1 & 1 & 1 & 1 & 1 & 1 & 1 \\
\hline & Nicandra physaloides & 1 & 1 & & 1 & 1 & 1 & 1 & 1 & 1 & 1 & 1 & 1 & 1 \\
\hline & Sahicea of hrasiliensis & 1 & 1 & & 1 & 1 & 1 & 1 & & 1 & 1 & 1 & 1 & 1 \\
\hline \multirow{4}{*}{$\operatorname{sPp} 6$} & Conyza canadensis & 1 & 1 & 1 & 1 & & & & & & 1 & 1 & 1 & 1 \\
\hline & cf. Gnaphalium spicatum & & 1 & & & 1 & & & & & & 1 & & \\
\hline & Hyptis pectinata & & 1 & & 1 & & & & & & & 1 & & \\
\hline & Portulaca oleracea & & 1 & & & & & & & & & 1 & & \\
\hline \multirow{4}{*}{$\operatorname{spp} 7$} & Cypl & & & & & & & & & & & 1 & 1 & 1 \\
\hline & Digitaria fuscescens & & & & & & & & & & & 1 & & \\
\hline & Neonotonia wightii & & & & 1 & & & & & & & & 1 & 1 \\
\hline & Tridax procumbens & & & & & & & & & & & 1 & & \\
\hline
\end{tabular}

$\mathrm{L}=$ lavoura contínua; $\mathrm{LPL}=$ lavoura-pastagem-lavoura; PLP = pastagem-lavoura-pastagem; $\mathrm{P}=$ pastagem contínua; $\mathrm{C}=$ preparo convencional do solo; $\mathrm{D}=$ semeadura direta; 1 = adubação de manutenção; 2 = adubação corretiva gradual; * $=P$. pedicelatum + P. polystachium + P. setosum. Os conjuntos florísticos SPP 3 e SPP 5 não foram inseridos na tabela. 
espécies que ocorreram em maior número de áreas: 9/10 e 8/10, respectivamente $O$ conjunto florístico SPP 4 caracterizou-se por agrupar espécies presentes no conjunto de todas as áreas (tanto em pastagens quanto em lavouras). Várias es pécies estão presentes no conjunto que agrupa as 13 áreas estudadas, e seis delas ocorreram em todas as áreas: Acanthospermumaustrale, Ageratum conyzoides, Eleusine indica, Richardia brasiliensis, Schwenckia americana e Solanum americanum. O conjunto florístico SPP 6 agrupou espécies características de áreas com baixo revolvimento do solo, e Conyzacanadensis (7/7) caracterizou esse conjunto. Em relação às espécies preferenciais encontradas, Murphy et al. (2006) também correlacionaram C. canadensis com campos sob semeadura direta. O conjunto florístico SPP 7 foi formado por espécies que ocorreram somente nas pastagens contínuas e nas áreas de cultivo de PLP com semeadura direta. Uma Cyperaceae (3/3) caracterizou esse conjunto. Verificou-se que 14 espécies ocorreram em apenas uma área.

As espécies consideradas características pelo TWINSPAN apresentaram menor importância em relação ao IVI, e as principais espécies de acordo com o IVI foram as que se encontraram em quase todas as áreas, estando entre as que estavam presentes no conjunto florístico 4 do TWINSPAN. Nesse caso, a identificação das condições mais favoráveis para as espécies com ampla distribuição foi efetuada pela análise dos resultados obtidos com o IVI. Ao avaliar as seis espécies mais importantes de cada área (Tabela 5), verificou-se que $B$. rhomboidea e $R$. brasiliensis foram importantes em áreas de lavoura com semeadura direta (LD1 e LD2), ao passo que Centratherum cf. camporum (LC1 e LC2), A. conyzoides e Digitaria cf. horizontalis (LC1, LC2, LPLC1 e LPLC2) tiveram maior importância em áreas com preparo do solo convencional. Cenchrus echinatus foi uma das espécies mais importantes na área de LC1, com 12,8\% de IVI, além de ter ocorrido em outras áreas com preparo con- vencional (LC2, LPLC1 e LPLC2), apresentando menor importância em áreas com semeadura direta (LD1, LD2 e LPLD1). De acordo com Pereira \& Velini (2003), houve redução na ocorrência de C. echinatus ao longo de quatro anos com a semeadura direta, o que poderia caracterizar uma condição desfavorável ao desenvolvimento dessa espécie.

Nas áreas com presença de forrageiras, foram importantes Sabicea cf. brasiliensis (LPLD2, PLPD1, PLPD2 e PC2), Eragrostis rufescens (LPLD1, PLPC 1, PLPD 1, PLPD2 e PC2) e S. americana (LPLD1, PLPC1, PLPD1, PLPD2 e PC2). Essas espécies são consideradas nativas do Cerrado (Mendonça et al., 1998). Segundo Nunes (2001), entre as principais espécies de infestantes que normalmente ocorrem nas pastagens na região do Cerrado encontramse essencialmente espécies nativas desse bioma. Nas áreas com adubação corretiva gradual, foram importantes Nicandra physaloides (LPLC2 e LPLD2) e S. americanum (LD2, LPLC2, LPLD2, PLPC2, PLPD2 e PC2) e, nas áreas com adubação de manutenção, Spermacoce cf. capitata (LPLD1 e PLPC1). De acordo com Kissmann (2000), N. physaloides prefere solos com boa fertilidade, assim como S. americanum. Segundo Kissmann \& Groth (2000), as espécies do gênero Spermacoce, em geral, conseguem se desenvolver em solos com menor fertilidade. $E$. indica esteve entre as seis espécies mais importantes em 11 das 13 áreas avaliadas. Essa espécie também esteve entre as principais espécies encontradas por Menezes \& Leandro (2004) em experimento com culturas de cobertura e preparo do solo em áreas de Cerrado. No entanto, E. indica ocorreu com menor importância em levantamento fitossociológico de duas áreas de pastagem (Tuffi Santos et al., 2004).

O sistema de cultivo e o de preparo do solo são os fatores mais importantes na determinação da estrutura florística dos bancos de sementes, enquanto a adubação corretiva gradual aumenta o número de famílias e de espécies em relação à adubação de manutenção. 
Tabela 5 - Índice de valor de importância (IVI) das principais espécies (negrito) encontradas em banco de sementes de 13 áreas de um experimento de cultivo lavoura-pastagem, na profundidade de 0 a $20 \mathrm{~cm}$ - Embrapa Cerrados, Planaltina-DF

\begin{tabular}{|c|c|c|c|c|c|c|c|c|c|c|c|c|c|}
\hline \multirow[b]{2}{*}{ Espécie } & \multicolumn{13}{|c|}{ Área } \\
\hline & త્ય & త్త & $\vec{\Xi}$ & $\hat{\mathrm{G}}$ & 岂 & $\underset{\Xi}{\mathcal{U}}$ & $\overrightarrow{\bar{a}}$ & 气ี & $\begin{array}{l}\vec{\Xi} \\
\vec{a}\end{array}$ & $\begin{array}{l}\text { U. } \\
a \\
a\end{array}$ & $\vec{a}$ & $\stackrel{\widehat{a}}{a}$ & ల̃ \\
\hline Acanthospermum australe & 9,8 & 2,1 & 6,6 & 0,9 & 11,0 & 2,2 & 9,2 & 1,6 & 20,6 & 8,5 & 41,3 & 5,6 & 3,2 \\
\hline Ageratum conyzoides & 50,5 & 48,1 & 26,4 & 39,2 & 54,5 & 68,4 & 5,2 & 24,4 & 12,5 & 47,7 & 6,0 & 9,6 & 7,2 \\
\hline Amaranthus cf. viridis & - & 2,0 & - & 1,1 & - & 2,4 & - & 8,9 & - & 1,9 & - & 1,7 & - \\
\hline Blainvillea rhomboidea & 4,3 & 7,1 & 9,0 & 8,6 & 2,0 & 3,2 & - & 5,7 & 1,8 & 1,9 & - & - & - \\
\hline Brachiaria decumbens & - & - & - & - & 1,0 & - & - & - & - & - & - & - & $\mathbf{5 7 , 0}$ \\
\hline Cenchrus echinatus & 12,8 & 9,2 & 4,8 & 5,7 & 3,0 & 2,2 & 2,0 & - & - & - & - & - & 3,2 \\
\hline Centratherum cf. camporum & 14,4 & 13,2 & 4,7 & 5,2 & 2,0 & - & 6,2 & 5,7 & - & - & - & - & - \\
\hline Commelina henghalensis & 1,1 & 12,1 & 1,0 & 8,2 & - & 4,6 & - & 1,6 & - & 2,3 & - & 1,9 & - \\
\hline Conyza canadensis & - & - & 1,3 & 1,8 & - & - & 7,3 & 3,2 & - & 3,6 & 4,0 & 11,2 & 14,2 \\
\hline Desmodium sp. & 4,4 & 6,0 & 8,1 & 16,7 & - & - & - & - & - & - & - & - & - \\
\hline Digitaria ciliaris & 9,4 & 9,4 & 2,2 & 6,1 & 11,2 & 7,9 & 6,1 & 2,2 & 9,3 & 3,1 & 1,8 & 1,6 & - \\
\hline Digitaria $\mathrm{cf}$. horizontalis & 23,0 & 14,6 & 8,5 & 4,4 & $\mathbf{3 6 , 3}$ & 12,7 & 3,9 & 3,8 & 3,7 & 1,6 & - & - & 3,2 \\
\hline Eleusine indica & 15,1 & 17,5 & 13,5 & 10,8 & 16,4 & 24,6 & 55,6 & 39,2 & 26,5 & 32,2 & 10,4 & 10,0 & 8,7 \\
\hline Eragrostis rufescens & 5,5 & 2,0 & 5,2 & - & 8,7 & 2,1 & 16,2 & 1,6 & 19,5 & 3,4 & 25,1 & 11,3 & 11,2 \\
\hline Nicandra physaloides & 2,0 & 8,2 & 1,1 & 5,4 & 3,0 & 11,0 & - & 23,5 & 2,8 & 3,1 & 2,2 & 9,8 & 4,7 \\
\hline Panicum maximum & 1,1 & - & - & - & - & - & - & - & 19,1 & 10,8 & 16,3 & 11,9 & - \\
\hline Pennisetum spp.* & 9,3 & 6,3 & 27,3 & 6,2 & 4,5 & 4,2 & 2,5 & - & - & 1,6 & - & - & - \\
\hline Rhynchelitrum repens & 6,4 & 1,8 & 9,7 & 5,9 & 4,0 & 2,1 & - & - & 7,7 & 1,6 & - & - & - \\
\hline Richardia brasiliensis & 18,2 & 11,4 & 32,4 & 19,6 & 12,5 & 11,3 & 16,5 & 3,2 & 8,5 & 9,9 & 12,6 & 33,8 & 4,7 \\
\hline Sabicea cf. brasiliensis & - & 1,8 & 1,9 & 2,8 & 2,0 & 1,1 & - & 11,9 & 6,8 & 1,9 & 17,8 & 20,7 & 40,9 \\
\hline Schwenckia americana & 2,3 & 1,8 & 2,9 & 4,4 & 1,1 & 3,3 & 9,3 & 6,8 & 23,4 & 7,2 & 45,3 & 44,0 & 7,2 \\
\hline Sida glaziovii & 1,1 & - & - & 1,9 & - & - & 20,6 & - & 1,8 & - & 2,2 & - & - \\
\hline Solanum americanum & 3,0 & 10,7 & 1,9 & 8,5 & 5,0 & 8,8 & 5,3 & 13,1 & 1,8 & 28,3 & 2,8 & 19,6 & 8,7 \\
\hline Spermacoce cf. capitata & 2,4 & 1,9 & - & - & 4,2 & 3,3 & 10,5 & 1,6 & 17,3 & 1,6 & 6,1 & - & - \\
\hline
\end{tabular}

$\mathrm{L}=$ lavoura contínua; $\mathrm{LPL}=$ lavoura-pastagem-lavoura; $\mathrm{PLP}=$ pastagem-lavoura-pastagem; $\mathrm{P}=$ pastagem contínua; $\mathrm{C}=$ preparo convencional do solo; $\mathrm{D}=$ semeadura direta; 1 = adubação de manutenção; 2 = adubação corretiva gradual; $*=P$. pedicelatum $+P$. polystachium + P. setosum.

\section{AGRADECIMENTOS}

Ao Institut de Recherche pour le Développement (IRD), Minis tère de Affaires Etrangeres, à Embrapa Cerrados, UnB e CAPES, pelo apoio no desenvolvimento deste trabalho.

\section{LITERATURA CITADA}

BALL, D. A.; MILLER, S. D. A comparison of techniques for estimation of arable soil seedbanks and their relationship to weed flora. Weed Res., v. 29, p. 365-373, 1989.
CARDINA, J.; HERMS, C. P.; DOOHAN, D. J. Crop rotation and tillage system effects on weed seedbanks. Weed Sci., v. 50, p. 448-460, 2002.

COBUCCI, T.; PORTELA, C. M. O. Manejo de herbicidas no sistema Santa Fé e na braquiária como fonte de cobertura morta. In: KLUTHCOUSKI, J.; STONE, L. F.; AIDAR, H. Integração lavoura-pecuária. Santo Antônio de Goiás: Embrapa Arroz e Feijão, 2003. p. 443-458.

FROUD-WILLIAMS, R. J.; CHANCELLOR, R. J.; DRENNAN, D. S. H. Potential changes in weed floras associated with reduced-cultivation systems for cereal production in temperate regions. Weed Res., v. 21, p. 99109, 1981. 
GASPARINO, D. et al. Quantificação do banco de sementes sob diferentes usos do solo em área de domínio ciliar.

R. Árvore, v. 30, n. 1, p. 1-9, 2006.

KENT, M.; COKER, P. Vegetation description and analysis: a practical approach. Chichester: John Wiley \& Sons, 1992. $363 \mathrm{p}$.

KISSMANN, K. G. Plantas infestantes e nocivas. 2. ed. São Paulo: BASF, 1997. Tomo I, 824 p.

KISSMANN, K. G. Plantas infestantes e nocivas. 2. ed. São Paulo: BASF, 2000. Tomo III, 722 p.

KISSMANN, K. G.; GROTH, D. Plantas infestantes e nocivas. 2. ed. São Paulo: BASF, 1999. Tomo II, 978 p.

LÉGÈRE, A.; STEVENSON, F. C.; BENOIT, D. L. Diversity and assembly of weed communities: contrasting responses across cropping systems. Weed Res., v. 45, p. 303-315, 2005.

MENDONÇA, R. C. et al. Flora vascular do cerrado. In: SANO, S. M.; ALMEIDA, S. P. Cerrado: ambiente e flora. Planaltina: Embrapa-CPAC, 1998. p. 289-556.

MENEZES, L. A. S.; LEANDRO, W. M. Avaliação de espécies de coberturas do solo com potencial de uso em sistema de plantio direto. Pesq. Agropec. Trop., v. 34, n. 3, p. 173-180, 2004.

MUELLER-DOMBOIS, D.; ELLENBERG, H. Aims and methods of vegetation ecology. New York: Wiley \& Sons, 1974. 547 p.
MURPHY, S. D. et al. Promotion of weed species diversity and reduction of weed seedbanks with conservation tillage and crop rotation. Weed Sci., v. 54, n. 1, p. 69-77, 2006.

NUNES, S. G. Controle de plantas invasoras cultivadas nos cerrados. Campo Grande: Embrapa Gado de Corte, 2001. (Documentos: Embrapa Gado de Corte, 117).

PEREIRA, F. A. R.; VELINI, E. D. Sistemas de cultivo no cerrado e dinâmica de populaçõ es de plantas daninhas.

Planta Daninha, v. 21, n. 3, p. 355-363, 2003.

SOSNOSKIE, L. M.; HERMS, C. P.; CARDINA, J. Weed seedbank community composition in a 35 -yr-old tillage and rotation experiment. Weed Sci., v. 54, n. 2, p. 263-273, 2006.

TUESCA, D.; PURICELLI, E.; PAPA, J. C. A long-term study of weed flora shifts in different tillage systems.

Leed Res., v. 41, p. 369-382, 2001.

TUFFI SANTOS, L. D. et al. Levantamento fitossociológico em pastagens degradadas sob condições de várzea.

Planta Daninha, v. 22, n. 3, p. 343-349, 2004.

VARGAS, L. et al. Emergência de plântulas de leiteiro (Euphorbia heterophylla L.) resistentes e sensíveis aos inibidores de ALS em diferentes profundidades de semeadura. B. Inf., Sociedade Brasileira da Ciência das Plantas Daninhas, v. 5, n. 1, p. 8, 1999.

VILELA, L. et al. Benefícios da integração lavoura-pecuária. In: KLUTHCOUSKI, J.; STONE, L. F.; AIDAR, H. Integração lavoura-pecuária. Santo Antônio de Goiás: Embrapa Arroz e Feijão, 2003. p. 143-170. 